\title{
Clinical Profile and Colonoscopic Findings in Patients with Ulcerative Colitis at a Tertiary Care Hospital in Nepal: a Cross Sectional Study
}

\author{
Subash Bhattarai*, Ramesh Raj Acharya \\ Department of Medicine, \\ Manipal College of Medical Sciences, Pokhara, Nepal.
}

Keywords: bloody diarrhea; colonoscopy; erosions; lower gastrointestinal bleed; ulcerative colitis

\section{Introduction}

Inflammatory Bowel Disease comprises conditions characterized by chronic or relapsing immune activation and inflammation within the gastrointestinal tract (GIT). ${ }^{1}$ Crohn's Disease (CD) and Ulcerative Colitis (UC) are the two major forms of IBD.

Ulcerative colitis is one of the common aetiology of lower GI bleed. The presenting symptom of Ulcerative Colitis are rectal bleeding, bloody diarrhea, tenesmus, chronic diarrhea with passage of mucus and crampy abdominal pain. ${ }^{2}$ Ulcerative Colitis is a condition in which the inflammatory response and morphologic changes remain confined to the colon. ${ }^{3}$ The rectum is involved in $95 \%$ of patients. ${ }^{4}$

The diagnosis is usually established through a global assessment of the clinical presentation, radiographic, colonoscopic and histological findings consistent with UC. Colonoscopy is an invasive procedure used for detection and management of various diseases of rectum, colon, and terminal ileum including ulcerative colitis. ${ }^{5}$ Direct visualization of pathological lesions and its use in taking tissue biopsies with confirmation by histology makes it investigation of choice for colonic pathology. Patients with long-standing ulcerative colitis are at increased risk for the development of intraepithelial neoplasia and carcinoma of colon. ${ }^{6,7}$

\begin{abstract}
Background and aims: Ulcerative Colitis (UC) is an inflammator condition confined to the colon. Ulcerative colitis commonly presents with rectal bleeding, bloody diarrhea and abdominal pain. This research was undertaken to study the clinical profile and colonoscopic findings of the patient presenting with ulcerative colitis in a tertiary care center at Gandaki Province,Nepal .
\end{abstract}

Methods: A hospital based prospective observational study was conducted from November 2017 till June 2020 in department of Medicine at Manipal teaching Hospital, Pokhara, Nepal after obtaining ethical approval from Institutional Research Committee and informed consent from patients or their relatives. Clinical profile and colonoscopic findings of patients with Ulcerative colitis were studied.

Results: Results: Out of 274 colonoscopies, 60 patients (M:F= 3: 2) were diagnosed with UC. The mean age of subjects was $37 \pm 3.56$ years. Chronic diarrhea and bloody diarrhea were the common presenting symptomps. Only proctitis (E1) was seen in $40 \%$, left sided colitis in $35 \%$ and pan colitis (E3) was observed in $25 \%$. Rectal involvement and erythema was observed in all. Loss of vascularity (96.7\%), erosions (93.3\%), increased granularity (85\%), followed by ulcers $(73.3 \%)$ and spontaneous bleed on touch $(50 \%)$ were the common findings in colonoscopy.

Conclusion: Lower Gl bleed is a common presenting manifestation in ulcerative colitis. Proctitis followed by left sided colitis was the common sites of involvement. Majority presented with disease of moderate severity. Most common features in UC patients were universal involvement of the rectum alongside erythema and erosions, loss of vascularity, increased granularity, followed by ulcers and spontaneous bleed on colonoscopy. 
Studies are scanty in this part of the country regarding ulcerative colitis. Considering above facts this research was undertaken to study the clinical profile and colonoscopic finding of the patient presenting with ulcerative colitis in a tertiary care center at Manipal Teaching Hospital in Pokhara, Gandaki Province, Nepal .

\section{Methodology}

This observational, cross sectional hospital based prospective study was carried out in the unit of Medical gastroenterology under Medicine department at Manipal College of Medical Sciences and Teaching Hospital, Gandaki Province, Nepal from November 2017 to June 2020 for a duration of 32 months after obtaining ethical clearance from Institutional Research Committee (MEMG/IRC/332/GA) and informed consent from patient or patient relatives.

The sample size was collected using the formula,

$\mathrm{n}=4 \times \mathrm{p} \times \mathrm{q} / \mathrm{e}^{2}$

Where, $p=$ prevalence

$$
\begin{aligned}
& q=1-p \\
& e=\text { margin of error }(0.05 \text { i.e. } 5 \%)
\end{aligned}
$$

From a previous study conducted in Nepal, prevalence of ulcerative colitis in patients undergoing colonoscopy was $10 \% .{ }^{8}$

So, sample size $\mathrm{n}=(4 \times 10 \times 90) / 25=144$

The calculated minimum sample size was 144. A total of 286 colonoscopies were performed in 30 months which was the sample size more than adequate for the study.

All patients, irrespective of age, sex or co morbidities who underwent colonoscopy for rectal bleed or haematochezia or bloody diarrhea or chronic diarrhea with abdominal pain were enrolled in the study. Patient with lower Gl bleed were first hemodynamically stabilized in the emergency or in ICU. Blood and blood products were transfused when required. History, physical examination and data considering demographic variables, clinical features, and symptomatology were recorded. Careful history on stool consistency, its frequency, presence of blood in stool etc and abdominal pain was taken. Data considering demographic variables, clinical features with fever, extra intestinal manifestations were documented. Blood investigations like complete blood count, platelets count, blood grouping, liver function test, prothrombin time/international normalized ratio (PT/ INR), coagulation profile were done. Ultrasonography of abdomen, CT abdomen was done when relevant. After taking proper consent, bowel preparation and pre medications, colonoscopy was performed. Bowel preparation was done with 2 liters of Poly Ethylene Glycol in patients with non active bleed. It was given 8 hours prior to procedure and patient kept on liquid diet from 24 hours prior to procedure. In case of acute emergency, only sigmoidoscopy after rectal enema was performed. A subset of patients who presented with acute flare up or active bleeding underwent unprepared sigmoidoscopy as well. During procedure patient was laid on left lateral position, intravenous Hyoscine butyl bromide was given. Each patient underwent endoscopic investigation by standard flexible colonoscope (PENTAX EPK 700, PENTAX JAPAN Inc). Colonoscopy findings were noted. Biopsy of tissue was sent for histopathology. The Truelove and Witt's score was used to classify severity of the disease (mild, moderate and severe). Montreal system of classification was used to describe the extent of the disease as E1 (limited to the rectum), E2 (distal to the splenic flexure) and E3 (extension proximal to the splenic flexure).

All patients who underwent colonoscopy for rectal bleed or haematochezia or bloody diarrhea or chronic diarrhea with abdominal pain were included. Patient with non specific colitis on colonoscopy and histology and patients with incomplete records or who fail to give informed consent were excluded from the study.

\section{Statistical Analysis:}

Data were collected covering the relevant parameters for the study. All categorical data were expressed in percent and absolute number. All numerical continuous data were expressed in mean \pm SD. The data analysis was done using Statistical Packages for the Social Sciences (SPSS) 20. (IBM Corp. Released 2011. IBM SPSS Statistics for Windows, Version 20.0. Armonk, NY: IBM Corp.)

\section{Results}

A total of 286 patients underwent colonoscopy from November 2017 till June 2020. But 12 patients had to be excluded because of inadequate data. Finally a total of 274 patients were taken up for the study. The most common finding was non specific colitis in $64(23.3 \%)$, followed by ulcerative colitis in 60(21.9\%), haemorrhoids in $36(13.1 \%)$ and polyps in $28(10.2 \%)$. Other findings were Chrons disease in $4(1.5 \%)$, colorectal cancer in $25(9.1 \%)$, diverticulum in $12(4.4 \%)$ and illeocecal tuberculosis in $62.2 \%)$ respectively. No abnormal findings were detected in $39(14.2 \%)$ colonoscopies.

Out of these 274 sample study, 64 patients were identified with IBD and 60 patients with ulcerative colitis. Only 4 patients had Chrons disease. The prevalence of ulcerative colitis was thus $21.9 \%$ in and around Gandaki Province in Nepal in the present study. Ulcerative colitis was 15 fold more common than Crohns disease. The mean age of these 60 subjects with ulcerative colitis was $37 \pm 3.56$ years with a range of $18-56$ years of age with $36(60 \%)$ males and 24 $(40 \%)$ females $(\mathrm{M}: \mathrm{F}=3: 2)$. Patients with ulcerative colitis were further classified as per sex and age groups with maximum of 26 (43.3\%) cases in 20-39 years of age group (Table 1 ).

\begin{tabular}{|c|c|c|c|c|c|}
\hline \multicolumn{5}{|c|}{ Table 1: Age groups / sex distribution of patients with } \\
ulcerative colitis & \multirow{2}{*}{ Total } \\
\hline \multirow{2}{*}{ SEX } & \multicolumn{5}{|c|}{ Age Groups } \\
\cline { 2 - 6 } & $<20$ yrs & $20-39$ yrs & $40-59$ yrs & $\geq 60$ yrs & \\
\hline Male & 4 & 15 & 11 & 6 & 36 \\
\hline Female & 2 & 11 & 9 & 2 & 24 \\
\hline
\end{tabular}

Chronic diarrhea in 57(95\%), blood diarrhea in 54(90\%), mucus in stool in 44(73.3\%), tenesmus in 38(63.3), abdominal pain in $32(53.3 \%)$ and frank, fresh rectal bleeding in $31(51.7 \%)$ were the most common presentation in these patients with ulcerative colitis. Ten (16.7\%) patients presented with shock with active lower GI bleed.

These subjects were classified according to their ethnicity/caste. Majority of subjects were found to belong from Mongols (32\%) followed by Chhetris (18\%), Brahmins (16.4\%), Newars (13.8\%) and Dalits (7.1\%). Others caste included $12.7 \%$ of patients. Majority of these patients were farmers (31.7\%) followed by retired personnel (28.4\%), job holders (23.2\%) and housewives (15.7\%). Majority $(54.3 \%)$ were from rural areas whereas rest $45.7 \%$ belonged to urban regions. Majority $(60 \%)$ of the patients were from middle socioeconomic status. 
Among the clinical signs, pallor was the most common finding in $80 \%$, followed by abdominal tenderness in $58.36 \%$, weight loss in $40 \%$, splenomegaly in $34 \%$, fever in $30 \%$ and hepatomegaly in $12.5 \%$. Clinically, $28(46.7 \%)$ patients had moderate disease. Nineteen (31.7\%) had mild disease and 13 (21.6\%) had severe disease as depicted in pie diagram 1 . Extra intestinal manifestations (EIM) in the form of arthralgia and arthritis were observed in $12(20 \%)$ patients and recurrent oral ulcers in $9(15 \%)$ patients.

Mean haemoglobin at presentation was $11.3 \mathrm{gm} \%$ (range of 6.2 gm\% to $13.5 \mathrm{gm} \%)$. Haemoglobin was $<10.5 \mathrm{gm} \%$ in $22(36.6 \%)$ subjects. Mean platelets count was $153,000 / \mathrm{mm} 3$ with a minimum of $82,000 / \mathrm{mm} 3$ and a maximum of $354,000 / \mathrm{mm} 3$. Liver function tests revealed hyperbilirubinemia in 19 (31.7\%) and increased ALT, AST, and prolonged Prothrombin time by more than $4 \mathrm{sec}$ in 22 (36.7\%), 19 (31.7\%), and 19(31.7\%) respectively. ESR was raised in 35 (58.3\%) patients. Average of 2.3 units of blood was transfused in 38 patients with active bleed or when haemoglobin was less than $10.5 \mathrm{gm} \%$. None of the patient required surgical intervention and their symptoms were usually controlled by blood transfusion, IV fluids and IV medications including IV steroids and oral mesalazine. No inpatient mortality was reported.

Complete colonoscopy with visualization of terminal ileum could be performed in only $51(85 \%)$ patients. Terminal ileum could not be intubated in 5 patients. Scope could not be negotiated beyond hepatic flexure in another subset of 4 patients.

Rectum was involved in all the cases. Twenty four (40\%) patients had proctitis extending up to recto sigmoid junction (E1). Left sided colitis (E2) was observed in 21 (35\%) patients and pan colitis (E3) was observed in $15(25 \%)$ patients as depicted pie diagram 2. Erythema was observed in all (100\%). Loss of vascularity in $58(96.7 \%)$, erosions in $56(93.3 \%)$ and increased granularity in $51(85 \%)$, followed by ulcers in $44(73.3 \%)$ and spontaneous bleed on touch in 30 (50\%) were the common findings in colonoscopy.

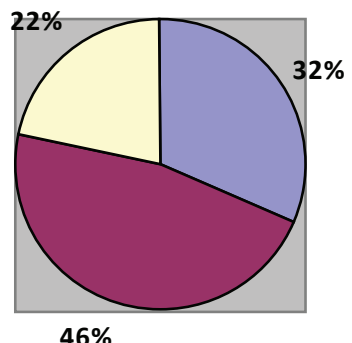

\begin{tabular}{|l|}
\hline$\square 19$ mild \\
$\square 28$ mod \\
$\square 13$ severe
\end{tabular}

$46 \%$

Pie Diagram 1: Severity of disease

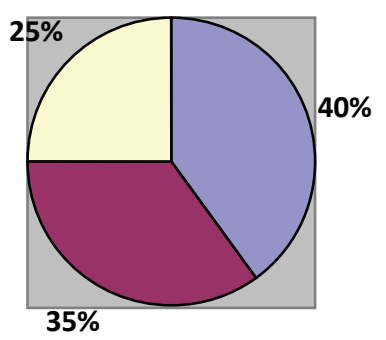

\begin{tabular}{|l|}
\hline$\square 24$ E1 Proctitis \\
$\square 21$ E2 Left colitis \\
$\square 15$ E3 Pancolitis \\
\hline
\end{tabular}

Pie Diagram 2: Extent of disease

All cases of ulcerative colitis were histologically proven. The most common findings were lymphoplasmacytic infiltration in all $(100 \%)$, glandular disruption in 58 (96.7\%), cryptitis in 53 (88.3\%) and crypt abscesses in $46(76.7 \%)$. Mild to moderate dysplasia was seen in $11(18.3 \%)$ cases but intra epithelial neoplasia or carcinoma was not detected.

\section{Discussion}

The prevalence of ulcerative colitis was $21.9 \%$ in the present study. Ulcerative colitis was seen in $10 \%$ colonoscopies in a previous study by Chaudhary et al. ${ }^{8}$ in Western Nepal. In a previous Nepalese study by Shrestha et $\mathrm{al}^{9}$, ulcerative colitis was seen in 10.4 $\%$ colonoscopies.. This suggests that the incidence of ulcerative colitis or its detection rate may be rising in Nepal. Ulcerative colitis was seen among $24 \%$ colonoscopies in the study by Hazare et al. ${ }^{11}$

In an Indian study by Islam et al. ${ }^{10}, 27$ patients were of Ulcerative colitis (UC) and 11 were of Chrons disease (UC:CD = 2.4:1). In the current study, presentation with Ulcerative colitis was even more compared to Chrons disease (UC: $C D=15: 1$ ).

In our current study, the mean age of subjects with ulcerative colitis was $37 \pm 3.56$ years with majority of cases in age group of 20-39 years of age with male predominance $(M: F=3: 2)$. The mean age of the patients was 38.04 years with male dominance in an earlier study by Pathak et al. ${ }^{12}$ in Nepal, which was almost similar to our findings. In the study by Islam et al, 10 majority of patients with ulcerative colitis were in the age group $20-39$ years with M:F $=2.9: 2$, findings similar to our study. Ulcerative colitis was frequent in younger age group below 50 years in the study by Alobaidi et al. ${ }^{13}$ and Shrestha et al. ${ }^{9}$ All these studies suggest that ulcerative colitis is commonly seen in young and middle age groups all over the world.

Majority of subjects were Mongols (32\%) followe by Chhetris, Brahmins and Newars in the current study whereas, majority (55\%) belonged to Brahmins in the study by Pathak et al. ${ }^{12}$ This reflects the disease is seen among all ethnicity within Nepal.

The common clinical presentations of patients in our study were chronic diarrhea in $95 \%$ and blood mixed stools in $90 \%$. Other findings were mucus in stool (73.3\%), tenesmus (63.3\%), abdominal pain (53.3\%) and frank, fresh rectal bleeding (51.7\%). Rectal urgency (100\%) followed by increased frequency of stools $(96.30 \%)$, blood in stools $(92.59 \%)$, mucus in stools $(88.89 \%)$, tenesmus (92.59\%) and loose stools (88.89\%) were the common presentations in the study by Islam et al. ${ }^{10}$ findings, almost similar to ours. Rectal bleeding (85\%) was more common than chronic diarrhea (70\%) in the study by Pathak et al. Rectal bleeding (80\%) was also the most common clinical feature in the study by Alobaidi et al. ${ }^{13}$

Haemoglobin of less than $10.5 \mathrm{gm} \%$ was observed in $36.6 \%$ subjects. Raised ESR and weight loss were seen in 58.3\% and $40 \%$ respectively in the current study. Weight loss and anemia were one of the key features of UC patients in the study by Chikhaliya et al. ${ }^{14}$ Blood investigation showed that $70 \%$ of the people had high ESR and $50 \%$ patients had anemia in the study by Meti et al. ${ }^{17}$

According to Silverberg et al. ${ }^{15}$, up to $25 \%$ of patients with UC can present with extra intestinal manifestations (EIM) during their lifetime. The most common EIM was arthralgia and arthritis (20\%) followed by recurrent oral ulcers (15\%) in our study. In the Indian study by Meti et al. ${ }^{16}, 39 \%$ of patients had extra intestinal manifestations and the most common was arthritis (16\%) The common extra intestinal manifestation were sacroiliitis in $31.9 \%$, mouth ulcers in $23.4 \%$, episcleritis in $8.6 \%$ and erythema nodosum in $2.1 \%$ in the study by Chikhaliya et al.14 More than one half of patients (UC 51.6\%) had one or more extra intestinal symptoms according to Makharia et al. ${ }^{17}$ Extra intestinal manifestations (EIM) were found among $12 \%$ of patients by Pathak et al. ${ }^{12}$

Nineteen (31.7\%) had mild disease and 13 (21.6\%) had severe disease in the current study. Majority comprising of 28 (46.7\%) UC patients were having moderate disease at presentation in the current study. It was similarly observed with $46 \%$ presenting with 
moderate severity in a previous Nepalese study by Pathak et al. ${ }^{12}$ Majority (59.26\%) of cases were also of moderate activity in the study by Islam et al. ${ }^{10}$ Pathak et al ${ }^{12}$ reported that $12 \%$ were having severe cases which was lesser when compared to our study.

Most common site of involvement was rectum and seen in all 100 $\%$ in the current study. Twenty four (40\%) subjects had proctitis extending up to recto sigmoid junction (E1). Left sided colitis (E2) was observed in 21(35\%) patients and pan colitis (E3) was observed in $15(25 \%)$ patients. Rectum was similarly involved in all the cases in the study by Islam et al. ${ }^{10}$ According to Pathak et al. ${ }^{12}$ in a previous Nepalese study, $41 \%$ had E1, higher compared to ours. Pan colitis was seen in $13 \%$ in the previous Nepalese study by Pathak et $\mathrm{al}^{12}$, lesser in comparison to ours. Contrary to above studies, Makharia et $\mathrm{al}^{17}$ reported that majority (42.8\%) had pan colitis followed by left-sided colitis in $38.8 \%$, and proctitis alone in $18.3 \%$ patients.

Rectal involvement and erythema in $100 \%$, loss of vascularity in $96.7 \%$, erosions in $93.3 \%$, increased granularity in $85 \%$ followed by ulcers in $73.3 \%$ and spontaneous bleed on colonoscopy in $50 \%$ were the common findings in colonoscopy. Involvement of rectum with erythema in $100 \%$ was similarly observed by Islam et al.10 Similar were the other findings; loss of vascular pattern $(85.19 \%)$, granularity and friability was seen in $81.48 \%$ of cases. ${ }^{10}$

Histology of the colonic mucosa suggestive of ulcerative colitis was evident with cryptitis in $88.3 \%$ and crypt abscesses in $76.7 \%$ in the current study. Mild to moderate dysplasia was seen in $18.3 \%$. Cryptitis was seen in $95 \%$ of the histology in the study by Meti et al. ${ }^{16}$ Dysplasia or atypia was detected in $9 \%$ whereas $2 \%$ of patients histology showed underlying carcinoma. ${ }^{16}$ No intraepithelial neoplasia or carcinoma was detected in any of our patients.

None of the patients in this study required surgical intervention and their symptoms were usually controlled by use of IV and oral medications. Similar were the findings by Chikhaliya et al. ${ }^{14}$ However, four percent of patients with UC had undergone colectomy in the study by Makharaia et al. ${ }^{17}$

\section{Limitation Of The Study}

This study had its own limitations. The study reflects to a certain geographical area. Sample size was small. Many patients lost to follow ups and follow up colonoscopies could not be performed in all patients. Due to lack of sedation and abdominal pain and distension perceived by the patients, illeo ceacal intubation was not possible in a minority of cases.

\section{Conclusion}

Chronic diarrhea, bloody diarrhea, bleeding per rectum , chronic abdominal pain and anemia were the common presentation of patients with ulcerative colitis. It was more common among males and in younger age groups. Pallor was the most common finding followed by abdominal tenderness, weight loss, splenomegaly, fever and hepatomegaly. Majority of patients presented with disease of moderate severity. Proctitis followed by left sided colitis was the common sites of involvement. Pan colitis was seen in $1 / 4$ th of the patients. Most common features were universal involvement of the rectum alongside erythema and erosions, loss of vascularity, increased granularity, followed by ulcers and spontaneous bleed on colonoscopy

\section{Recommendation}

Colonoscopy or at least flexible sigmoidoscopy should be offered when the patients complain of fresh rectal bleeding or bloody diarrhea or chronic diarrhea and rule out causes like ulcerative colitis, colon polyps or colorectal carcinomas. This should be followed by histological assessment of tissue biopsies.

\section{Acknowledgement}

The authors take this opportunity to thank all the endoscopy staffs of Manipal Teaching Hospital.

\section{Conflict of Interest}

None

Source of funding

None

\section{References:}

1. Hendrickson BA, Ranjana Gokhale and Judy H. Cho Clinical aspects and pathophysiology of Inflammatory Bowel Disease: Clin Microbiol Rev.2002;15:79-94

2. Cosnes J, Gower-Rousseau C, Seksik P, Cortot A. Epidemiology and natural history of Inflammatory Bowel Diseases. Gastroenterology. 2011;140(6):1785-94.

3. Loftus E V. Epidemiology of Inflammatory Bowel Disease. In: Gl Epidemiology: Diseases and Clinical Methodology: SecondEdition. 2014. p. 273-84.

4. Satsangi J, Silverberg MS, Vermeire S, Colombel JF. The Montreal classification of inflammatory bowel disease: controversies, consensus, and implications. Gut. 2006; 55(6):749-53.

5. Rex DK, Petrini JL, Baron TH, Chak A, Cohen J, Deal SE et al. Quality indicators for colonoscopy, Gastrointest Endosc. 2006; 63 (4):16-28.

6. Ekbom A, Helmick C, Zack M and Adami HO. Ulcerative colitis and colorectal cancer. A Population-Based Study. N. Engl. J. Med.1999; 323(18):1228-33.

7. Sebastian S, Hernández V, Myrelid P, Kariv R, Tsianos E, Toruner $M$ et al. Colorectal cancer in inflammatory bowel disease: Results of the 3rd ECCO pathogenesis scientific workshop (I). Journal of Crohn's and Colitis. 2014 ; 8(1):5-18

8. Chaudhary, S, Chaudhary, P, Jaiswal N and Chaurasia R. Colonoscopy: A Two Year Experience from Western Nepal. Journal of Universal College of Medical Sciences. 2013; 1(3): 28-32.

9. Shrestha UK. Etiological profile, gender difference and age group patterns of 415 patients presenting with lower gastrointestinal bleeding in the western region of Nepal. Journal of Advances in Internal Medicine 2014; 3(2):52-55.

10. Islam S, Agarwal M, Talukdar A J, Dutta S, Kalita PP. A study on clinical profile of patients with Inflammatory bowel disease. National journal of medical research.2016: 6(3):233-38

11. Hajare S, Kantamaneni R. Etiological profile of patients with lower gastrointestinal bleeding: A 1-year cross-sectional study. Arch Med Health Sci. 2018; 6(2):300-02.

12. Pathak R, Sherpa TW, Jha A, Hamal R, Thapaliya S. Sociodemographic and clinical characteristics of patients with Ulcerative Colitis at a Tertiary Care Centre in Nepal. JAIM. 2019; 8(2):26-29

13. Alobaidi QA, Al-shammari A J, Al- Faham AM. Colonoscopy 
finding of lower gastrointestinal bleeding (LIGB) in AlSeder Medical City (in Al-Najaf)/Iraq. J. Pharm. Sci. \& Res. 2018:10(4):839-42

14. Chikhaliya DP, Patel PG, Patel RP, Mehta KV. Demographic Profile and Clinical Presentation of Ulcerative Colitis in Trimurti Hospital from 2007 to2014. EC Gastroenterology and Digestive System. 2017; 3(1): 14-22.

15. Silverberg MS, Satsangi J, Ahmad T, Arnott ID, Bernstein CN, Brant SR. Toward an integrated clinical, molecular and serological classification of inflammatory bowel disease: report of a Working Party of the 2005 Montreal World Congress of Gastroenterology. Can J Gastroenterol. 2005 ; 19 Suppl A:5A$36 \mathrm{~A}$

16. Meti BS, George P, Stany Al. Clinical profile of patients with Ulcerative colitis at a tertiary care hospital, Mangaluru, India.
International journal of scientific research. 2019; 8(9):47-48

17. Makharia GK, Ramakrishna BS, Abraham P, Choudhuri G, Misra SP, Ahuja $V$ et al. Survey of inflammatory bowel diseases in India. Indian Society of Gastroenterology Task Force on Inflammatory Bowel Disease. Indian J Gastroenterol. 2012 ;31(6):299-306. 